\title{
The impact of bilingualism on the executive control and orienting networks of attention*
}

\author{
MIREIA HERNÁNDEZ \\ Departament de Psicologia Bàsica, Universitat de \\ Barcelona, Spain \\ ALBERT COSTA \\ ICREA \& Departament de Tecnologies de la Informació \\ i les Comunicacions, Universitat Pompeu Fabra, \\ Barcelona, Spain \\ LUIS J. FUENTES \\ Universidad de Murcia, Spain \\ ANA B. VIVAS \\ City Liberal Studies: Affiliated Institution of the \\ University of Sheffield, Thessaloniki, Greece \\ NÚRIA SEBASTIÁN-GALLÉS \\ Departament de Tecnologies de la Informació i les \\ Comunicacions, Universitat Pompeu Fabra, Barcelona, \\ Spain
}

(Received: March 9, 2009; Revised: April 2, 2009; Accepted: April 3, 2009; First published online 4 May 2010)

\begin{abstract}
The main objective of this article is to provide new evidence regarding the impact of bilingualism on the attentional system. We approach this goal by assessing the effects of bilingualism on the executive and orienting networks of attention. In Experiment 1, we compared young bilingual and monolingual adults in a numerical version of the Stroop task, which allowed the assessment of the executive control network. We observed more efficient performance in the former group, which showed both reduced Stroop Interference and larger Stroop Facilitation Effects relative to the latter. Conversely, Experiment 2, conducted with a visual cueing task in order to assess the orienting network, revealed similar Cueing Facilitation and Inhibition (Inhibition of Return - IOR) Effects for both groups of speakers. The implications of the results of these two experiments for the origin and boundaries of the bilingual impact on the attentional system are discussed.
\end{abstract}

\section{Introduction}

Previous research has provided positive evidence regarding an effect of bilingualism on cognitive control processes (e.g., Bialystok, Craik, Klein and Viswanathan, 2004; Bialystok, Martin and Viswanathan 2005a; Bialystok, Craik, Grady, Chau, Ishii, Grunji et al., 2005b; Bialystok, 2006; Martin-Rhee and Bialystok, 2008; Costa, Hernández and Sebastián-Gallés, 2008; Costa, Hernández, Costa-Faidella and Sebastián-Gallés, 2009). These advantages are usually attributed to the processes involved in what is often referred to as the executive control network of attention (see below), and are usually indexed by the better performance of bilinguals in tasks that require conflict resolution (e.g., Simon and flanker tasks). In these tasks, adult bilinguals are usually

* This research was supported by three grants from the Spanish Government (SEJ-2005/SEJ2005-01223/CONSOLIDER-INGENIO) and grants PSIC and PSI2008-00464/PSIC, as well as by the "ICREA Academia" prize for excellence in research awarded by the Generalitat de Catalunya to N.S.G. Mireia Hernández was supported by a predoctoral fellowship from the Catalan Government. The authors are grateful to Dr. Bialystok, Ms. Ivanova and Ms. Aalia Chatur for their comments on previous versions of this manuscript. faster (e.g., Bialystok et al., 2004; Bialystok et al., 2005a; Bialystok, 2006; Costa et al., 2008; Martin-Rhee and Bialystok, 2008), and/or they show a reduced conflict effect (Bialystok et al., 2004; Bialystok, Craik and Luk, 2008; Costa et al., 2008). Although little is known about the origin of the bilingual advantage, researchers often relate it to the cognitive mechanisms involved in bilingual language control. That is, the fact that bilinguals need to choose between two alternative representations (a word and its translation) may engage some of the same cognitive and brain mechanisms that are involved in the domaingeneral executive control system (e.g., Green, 1998; Bialystok et al., 2004; Abutalebi and Green, 2007, 2008; Kroll, Bobb, Misra and Guo, 2008).

Despite these observations, concerns have been raised regarding the reliability and replicability of the bilingual advantage, especially in the case of young adults that are at the peak of their attentional capacities (Morton and Harper, 2007; Colzato et al., 2008). For example, Colzato et al. rightly pointed out failures to replicate the bilingual advantage in conflict resolution (e.g., Bialystok et al., 2005a). Thus, it is still important to bring new, robust evidence about the attentional effects of bilingualism in young adults. 
Granting the presence of a bilingual advantage, we still need to determine its boundaries. There are at least two ways to proceed. First, we need to identify which components of the theoretical construct called executive control are affected by bilingualism. This is a difficult task since there does not seem to be a unified view regarding these processes, and researchers often merely relate them to specific task demands (e.g., Bialystok, 2006). However, progress might be made by using an experimental approach that explores the effects of bilingualism in different experimental paradigms. This strategy will hopefully enable robust empirical generalizations, eventually leading to the identification of the specific processes that are responsible for the bilingual advantage. The second avenue is to explore the extent to which bilingualism affects other attentional processes outside the executive control network. Given that some cross-talk (see below) seems to exist between the processes involved in different attentional components, it is conceivable that bilingualism actually exerts an effect beyond the executive control network. The present article takes both of these approaches. First, we assess the effects of bilingualism in young adulthood in a task that has not yet been explored (Experiment 1) and that is supposed to involve some of the processes in which bilinguals excel (conflict monitoring and resolution). Second, we explore the presence of a bilingual effect on a task that indexes the functioning of processes outside executive control (Experiment 2).

In the following, a brief overview of a general framework of the attentional system is presented, followed by a discussion of the potential effects of bilingualism on the orienting network of attention.

\section{Components of the attentional system}

One of the most influential frameworks regarding the organization of the attentional system is that proposed by Posner and collaborators (Posner and Boies, 1971; Posner and Petersen, 1990). According to this framework, the attentional system can be fractionated in three different cognitive and anatomical networks sustaining different cognitive abilities: the alerting, the orienting and the executive networks. The executive network is mainly sustained by the anterior cingulate cortex (ACC) and bilateral frontal areas. The brain areas involved in the orienting network are parietal sites and frontal eye fields. Finally, the alerting network is more distributed in the brain, across the thalamus and both the anterior and posterior cortical sites (e.g., Fan, McCandliss and Fossella, 2005).

From a cognitive point of view, the executive network groups together those processes involved in driving the behavior of the individual in a goaloriented manner. This network will be mostly involved in planning goals, developing strategies, sustaining goals and adjusting them to novel context demands when needed, while avoiding distracting information irrelevant to the desired objective (Posner and Boies, 1971). These abilities are often explored by means of tasks that require switching goals, resolving conflict between two incompatible responses, avoiding response to salient stimuli, withholding preponderant responses, etc. (e.g., Stroop, 1935; Simon and Rudell, 1967; Fan, McCandliss, Sommer, Raz and Posner, 2002; Barceló, 2003). Perhaps because we lack a unitary account of the functioning of the executive control network, our knowledge about the specific processes that are affected by bilingualism is rather limited. Nevertheless, the bilingual advantage on executive control has been linked to the efficiency of inhibitory control processes. In fact, various models of bilingual speech production posit that selection of the target word in the desired language involves the inhibition of the competing translation words (Green, 1986, 1998; Meuter and Allport, 1999; Lee and Williams, 2001; Costa, Santesteban and Ivanova, 2006; Kroll, Bobb and Wodniecka, 2006; Kroll et al., 2008; but see Roelofs, 1998; Costa and Caramazza, 1999; Costa, Miozzo and Caramazza, 1999; Finkbeiner, Gollan and Caramazza, 2006). However, further studies are required in order to advance in our understanding of how bilingualism affects the executive control network.

The orienting network is mainly involved in the allocation of cognitive resources towards potentially relevant stimuli according to their specific sensory properties (e.g., unique features, novelty or abruptness; see Ruz and Lupiáñez, 2002). In a way, the processes implicated in this network are mostly driven by bottomup environmental stimuli surrounding the individual, rather than by internally generated goal-oriented factors. Finally, the alerting network leads to the achievement and maintenance of the alert state necessary for quick detection of the expected stimulus.

Although these three networks are anatomically and functionally distinct, they work in an orchestrated manner. For example, the efficiency of the processes involved in ignoring potentially distracting information (executive control network) is affected by the alerting level (alert network); if the alert level is high, ignoring distracting information appears to be harder (see footnote 1). Along the same lines, the processes involved in the executive function may be affected by orienting attention to the relevant stimuli (the resolution of the conflict created by distracting information seems to be better when attention is oriented towards the experimental stimulus). ${ }^{1}$

1 Experimental evidence of the cross-talk between the three attentional networks comes mainly from studies by Callejas and collaborators (Callejas, Lupiáñez and Tudela, 2004; Callejas, Lupiáñez, Funes and Tudela, 2005) in which a task requiring conflict resolution (executive network) is combined by auditory (alerting network) and visual (orienting network) cues. These mutual influences consist of at least three different modulations between networks. First, when combining 
Considering this cross-talk between the networks, one may think that external factors affecting one of them might percolate to the others. Hence, it is conceivable that bilingualism, by affecting the functioning of the executive control network, may somehow modify the functioning of other attentional processes. In fact, there is at least one empirical observation supporting this view. In Costa et al. (2008), bilinguals appeared to have larger alerting effects than monolinguals. That is, bilinguals benefit more (responded faster to the target) than monolinguals upon the presentation of an alerting cue. ${ }^{2}$ In Experiment 2, we assess whether this cross-talk between attentional networks may actually lead to an effect of bilingualism on the orienting network of attention.

\section{The bilingual effect on the orienting network}

The available experimental evidence regarding the effects of bilingualism on the orienting network of attention is contradictory. Let us discuss this evidence in detail.

In a recent study, Colzato et al. (2008, Experiment 2), explored the effect of bilingualism on the visual cueing effects in target detection. In this task, participants were asked to detect a target that could appear above or below a fixation point. The target was preceded, at different Stimulus Onset Asynchronies (SOAs), by a visual cue that appeared in the same (valid cue) or opposite location (invalid cue). In this setting, it is possible to calculate the effect on response times of valid and invalid cues. Interestingly, whether or not a valid cue facilitates or hampers the detection of a target stimulus (always in comparison to an invalid cue) depends on the SOA between cue and target. When a valid cue is presented very shortly before the target, target detection is facilitated in comparison to when the cue is invalid - the cueing facilitation effect. In contrast, when the SOA increases, valid cues delay target detection in comparison to invalid cues (the Inhibition of Return effect - IOR). Colzato's study explored whether these two cueing effects were modulated by bilingualism. The authors predicted that

a flanker task paradigm with an alerting signal, Callejas et al. (2004, 2005) observed a larger conflict effect in those trials where an alerting signal was previously presented. Second, the same authors found that the orienting network exerts a positive influence on the executive one. Specifically, they observed that the conflict effect was smaller in those trials in which a visual cue indicated the location of the upcoming target (valid cues) than in those trials in which a visual cue was presented at the opposite location of the upcoming target (invalid cues). Third, the alerting network improves the efficiency of the orienting one. That is, the orienting effect is bigger when an alerting tone had been presented before the visual valid cue than in those visually cued trials without a previous alerting tone (Callejas et al., 2004, 2005; Fuentes and Campoy, 2008).

2 At present we do not have a clear explanation of how this effect comes about, and further research needs to be conducted to assess its reliability. "although both groups should show comparable performance at short SOAs ... bilinguals should show a more pronounced IOR at longer SOAs that monolinguals do" (p. 305). This predicted pattern was not found. However, there was a very interesting observation in this study: bilinguals and monolinguals were affected differently by valid and invalid cues as indexed by the interaction between Type of Cue and Group of Participants, leading the authors to conclude that "it is clear that cueing effects were affected by bilingualism" (p. 307).

The way in which bilingualism affected the cueing effects in Colzato et al.'s study was unfortunately complex. At short SOAs (100 ms), cueing facilitation effects were found only for monolinguals. Second, at long SOAs (700 ms), IOR effects were present only for bilinguals.

The cueing facilitation effects are at odds with previous results by Costa et al. (2008). In that study, conducted with 200 participants, we did not observe any impact of bilingualism on visual cueing effects. Unlike Colzato et al.'s study, in which cueing facilitation effects were present only in monolingual speakers $(25 \mathrm{~ms}$ for monolinguals and $8 \mathrm{~ms}$ for bilinguals), in Costa et al.'s study these effects were identical for both groups $(76 \mathrm{~ms}$ for monolinguals and $80 \mathrm{~ms}$ for bilinguals).

Given these contrasting results, the conclusion reached by Costa et al. (2008) may be premature. Unfortunately, a direct comparison between these two studies is difficult because of the many differences between the experimental designs used. For example, the two studies differ in the number of SOAs used (four vs. one), the task that participants had to perform (detect the inclination of a line vs. detect the direction of an arrow), and even the engagement of conflict resolution processes (absence vs. presence of conflict stimuli). If anything, the design of Colzato et al. appears to be more suited for exploring the cueing effects, and consequently one may take their observations as positive evidence of the effects of bilingualism on the orienting network.

The present study has two main goals. First, we aim at providing new evidence of the effect of bilingualism on executive control processes (Experiment 1). This will enable alleviation of the concerns raised by several authors about the replicability of such observations in young adults, and will also help to assess the generalization of the phenomenon itself. Second, we aim to assess the boundaries of the bilingual advantage in attentional processes by testing whether bilingualism impacts the processes involved in the orienting network of attention (Experiment 2).

\section{Experiment 1. Does bilingualism exert an effect on executive control?}

The aim of this experiment is to examine the impact of bilingualism on executive control by means of a 
Table 1. Means of reaction times (RTS) and percentage of errors (\% errors) as a function of Distractor (congruent, neutral and incongruent) and Group of Participants (bilinguals and monolinguals). Values in brackets refer to standard errors.

\begin{tabular}{llllllll}
\hline \hline & \multicolumn{3}{c}{ RTs } & & \multicolumn{3}{c}{$\%$ errors } \\
\cline { 2 - 3 } \cline { 8 - 9 } Group & Congruent & Neutral & Incongruent & & Congruent & Neutral & Incongruent \\
\hline Bilingual & $508(57)$ & $547(59)$ & $573(66)$ & & $1.13(1.98)$ & $1.74(2.53)$ & $6.88(3.54)$ \\
Monolingual & $543(88)$ & $570(86)$ & $610(98)$ & & $0.95(1.6)$ & $1.83(3.02)$ & $6.01(3.91)$ \\
\hline \hline
\end{tabular}

numerical version of the Stroop task (Stroop, 1935). In this context, it is important to note the existence of some recent evidence revealing that bilingualism may reduce the color-word Stroop effect (Bialystok et al., 2008). Importantly, however, the version of this Stroop paradigm included linguistic material (the classical colorword interference). It is possible then that the bilingual advantage in this task reveals just a better control of linguistic representations by bilingual speakers, rather than a general effect of bilingualism on executive control processes. Our study complements and extends this previous study by exploring the effect of bilingualism on a version of the Stroop task that does not involve linguistic material. It is also relevant to point out that, unlike in most of the previous studies that have addressed the bilingual impact on conflict resolution tasks, in this experiment the conflicting dimensions are not spatial in nature (e.g., arrow flanker task, or Simon task) but they are relatively more iconic (number and magnitude of the items). This manipulation allows us to extend the potential bilingual advantage to a conflict resolution task in which the source of the conflicting information is different from the one previously studied, therefore helping to build a body of evidence upon which to draw robust empirical generalizations.

Of special interest in this experiment is the interaction between the Stroop interference effect (SIE) and the Group of Participants. That is, an effect of bilingualism in this task will be indexed by an interaction between the factor Stroop interference effect $^{3}$ (neutral vs. incongruent trials) and Group of Participants (bilingual, monolingual).

3 We use the neutral condition in order to calculate the magnitude of the Stroop interference effect, rather than the congruent condition, because of the potential effects of bilingualism on the congruent condition as well. In fact, the performance of bilinguals and monolinguals was comparable in the neutral condition, but not in the congruent or incongruent conditions, where bilinguals in comparison to monolinguals were significantly faster. Hence, it appears that the neutral condition is the most appropriate one to explore the Stroop interference and facilitation effects, at least in this study (see also Bialystok et al. (2008) for a similar argument).

\section{Method}

\section{Participants}

Eighty-two participants performed the experiment (41 Catalan-Spanish bilinguals: 37 women and 4 men, ranging in age between eighteen and thirty years; and 41 Spanish monolinguals: 31 women and 10 men ranging in age between seventeen and twenty-nine years) (see Appendix for a detailed description of the participants in all experiments).

\section{Design and procedure}

Participants were asked to indicate, as fast and accurately as possible, how many items appeared in each trial. The number of items ranged from 1 to 3 , and participants had to press the keys 1, 2 or 3 on the keyboard with the index, middle and ring fingers of their dominant hand, respectively. There was a Distractor variable with three conditions: (a) alphabetic characters (neutral condition: e.g., Z, GGG, MM); (b) digits whose value matched the number of items (congruent condition: 1, 22, 333); and (c) digits whose value did not match the number of items (incongruent condition: e.g., 2, 33, 111, etc.). The experiment consisted of two blocks of 84 trials each (preceded by a training block of 24 trials). The three distractor conditions were represented the same number of times in each block in a random fashion. On each trial, a central fixation cross appeared for $1000 \mathrm{~ms}$, immediately followed by the target, which was presented for $2000 \mathrm{~ms}$ or until a response was given.

\section{Results and discussion}

Latencies faster than $250 \mathrm{~ms}$ or slower than $1500 \mathrm{~ms}$ were excluded from the analyses (.55\% of the trials). An ANOVA with Distractor (neutral, congruent, incongruent) as a within-subject variable, and Group of Participants (monolingual vs. bilingual) as a between-subject variable was conducted. In the error analyses, the only significant main effect was that of Distractor $(F(2,160)=148.34$, $M S E=4.74, p<.0001)$, revealing more errors in the incongruent $(6.45 \%)$ than in the neutral $(1.79 \% ; t(81)=$ $13.57, p<.0001)$ and congruent $(1.05 \% ; t(81)=13.11$, 


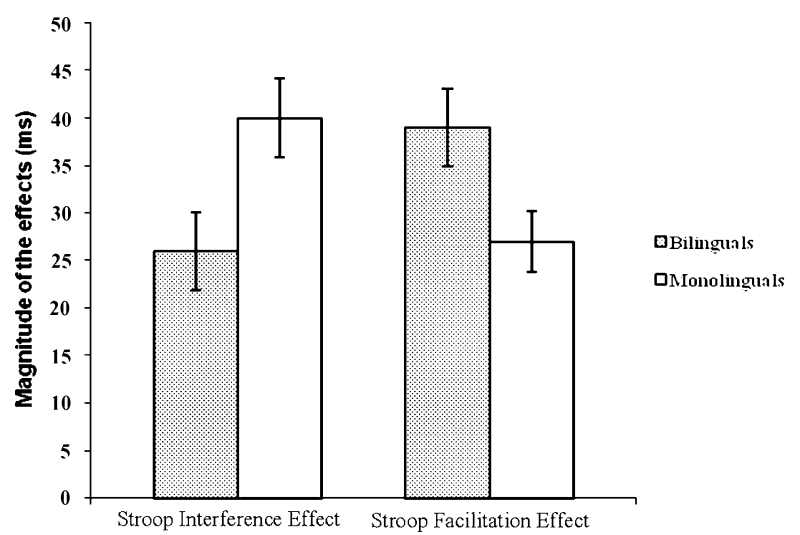

Figure 1. The Stroop Interference Effect is calculated by subtracting responses to "neutral trials" from those to "incongruent trials". The Stroop Facilitation Effect is calculated by subtracting responses to "neutral trials" from those to "congruent trials". Error bars represent standard error.

$p<.0001)$ conditions. Also, the neutral condition elicited more errors than the congruent one $(t(81)=3.06$, $p<.003$ ) (see Table 1).

In the analyses of reaction times (RTs), the main effect of Distractor was significant $(F(2,160)=253.62$, $M S E=350.79, p<.0001)$; the responses in the neutral condition were $33 \mathrm{~ms}$ faster than in the incongruent condition (the SIE; $t(81)=11.06, p<.0001$ ) and 33 $\mathrm{ms}$ slower than in the congruent condition (the Stroop facilitation effect $-\mathrm{SFE} ; t(81)=12.27, p<.0001)$.

Bilingual participants had a tendency to be faster (543 ms) than monolinguals $(574 \mathrm{~ms})$ (Group of Participants: $F(1,80)=3.59, M S E=17248.99, p=.061)$. Interestingly, the interaction between Distractor and Group of Participants was significant $(F(2,160)=3.48$, $M S E=350.79, p<.05)$. As this interaction suggests differences between the groups in either the SIE or the SFE (or both), we conducted two separate ANOVAs for each of these effects, including the RTs of the relevant conditions of the Distractor factor as a within-subject variable and the Group of Participants as a between-subject variable. The main effect of the "SIE" (incongruent vs. neutral condition) was significant $(F(1,80)=130.19, M S E=$ $346.77, p<.0001)$. More importantly, the SIE interacted with the variable Group of Participants, revealing that its magnitude was smaller for bilinguals $(26 \mathrm{~ms})$ than for monolinguals $(40 \mathrm{~ms} ; F(1,80)=6.14, M S E=346.76$, $p<.015$ ). The main effect of the SFE (congruent vs. neutral condition) was also significant $(F(1,80)=158.82$, $M S E=275.96, p<.0001)$. Interestingly, this effect also interacted with the factor Group of Participants, revealing that its magnitude was larger for bilinguals (39 ms) than for monolinguals $(27 \mathrm{~ms} ; F(1,80)=5.37, M S E=275.96$, $p<.023$ ) (see Figure 1).
The present experiment revealed three main results. First, bilinguals showed a reduced SIE in comparison to monolinguals, revealing a bilingual advantage in conflict resolution. Second, we also observed a larger SFE for bilingual speakers. Third, bilinguals appear to be somewhat faster than monolinguals across all experimental conditions.

The bilingual advantage in resolving conflict produced by irrelevant information (indexed by the SIE) is consistent with the bilingual advantage observed in other tasks - Simon and flanker tasks (e.g., Bialystok et al., 2004; Costa et al., 2008). In particular, our results extend the observation of Bialystok et al. (2008) to experimental conditions in which, arguably, very little linguistic processing is present. The bilingual advantage when the irrelevant dimension is congruent with the required response (indexed by the SFE) is also in line with Bialystok et al. (2008), who found larger SFE in bilinguals in the classical Stroop color-naming task. This latter observation suggests that the impact of bilingualism is not limited to conditions in which inhibitory processes (either active or reactive) are engaged (see "General Discussion").

We also found a tendency for bilinguals to be faster overall than monolinguals. As discussed in the "Introduction", this is a rather common finding and has been taken as indexing a bilingual advantage in the monitoring processes put at play in tasks that involve different types of stimuli. ${ }^{4}$

\section{Experiment 2: Does bilingualism aid orienting processes of attention?}

In this experiment we assess whether bilingualism influences the orienting network of attention by exploring the effects of valid and invalid visual cues on target detection. Participants were asked to decide whether a target stimulus was a straight line or a tilted one. The target could appear above or below a fixation point. Before the target, a visual cue was presented, either at the same position as the target (valid cue) or at the opposite position (invalid cue). By subtracting responses in these

4 The overall difference in RTs between monolinguals and bilinguals might compromise an interpretation of the differences between the two groups in the magnitude of the Stroop interference effects. Arguably, the faster the participants are the smaller the effects might be. However, in our experiment this is not always the case, since the Stroop facilitation effect is larger for the faster group (bilinguals). Furthermore, the magnitude of the Stroop interference effect did not correlate with overall reaction times within the group of bilingual speakers $(r=.22)$. In other words, it is not the case that the faster the participant is the smaller the Stroop interference effect is. At any rate, further research needs to clarify not only the origin of the overall advantage in reaction times associated with bilingualism, but also how such an effect might interact with the ability to resolve response conflict. 




Figure 2. Schematic representation of the events in a trial of Experiment 2. The example represents a trial with a valid cue.

two conditions, several cueing effects can be observed, depending on the time interval between cue and target. When the cue is presented shortly before the target, valid cues lead to faster RTs (the cueing facilitation effect). However, when the time interval between cue and target increases, responses to trials preceded by a valid cue start to get slower than those to trials preceded by an invalid cue (the IOR effect).

An effect of bilingualism on the functioning of the orienting network will be primarily indexed by an interaction between the factors Type of cue (valid vs. invalid) and Group of Participants (bilinguals and monolinguals). In fact, this interaction was significant in Colzato et al.'s (2008) Experiment 2 (see "Introduction"). Thus, following their arguments, we will take the presence of such an interaction as indexing differential cueing effects in both groups of participants. To the extent that cueing effects reveal the functioning of the orienting network, the presence of such an interaction should be taken as positive evidence of the effects of bilingualism on this network.

\section{Method}

\section{Participants}

Fifty-six participants completed the experiment (28 Catalan-Spanish bilinguals: 24 women and 4 men, mean age twenty years; and 28 Spanish monolinguals: 23 women and 5 men, mean age twenty-one years).

\section{Design and procedure}

The design and procedure were identical to that used by Colzato et al.'s (2008) Experiment 2. Each trial started with three boxes, one in the center of the screen and one above and below. This display was present for $1000 \mathrm{~ms}$. Thereafter, one of the peripheral boxes became thicker for $50 \mathrm{~ms}$ (the peripheral cue). Subsequently, the two peripheral boxes and the central fixation remained on the screen with the same thickness for $50,350,650$ or $950 \mathrm{~ms}$, depending on the SOA, and were followed by the target. The effective SOAs were therefore 100, 400, 700 and $1000 \mathrm{~ms}$ and these were randomized across trials. The target consisted of a white line $(2.6 \times 3.0 \mathrm{~cm}$ from a viewing distance of $60 \mathrm{~cm}$ ), and could appear either inside of the top or bottom box. The target appeared for $33 \mathrm{~ms}$, and right after there was a display with three boxes and the fixation cross, which was present until response or until $2000 \mathrm{~ms}$ had elapsed. Participants had to indicate the orientation of a line (horizontal or vertical), by pressing the key A or L. The mapping keyto-response was counterbalanced across participants. If participants made a mistake there was a beep, and they were instructed to be as fast as possible but without making mistakes. We also instructed participants to keep their eyes fixated at the center throughout the experiment (see Figure 2).

There were one practice block and eight experimental blocks of 40 trials each. Cues and targets were presented an equal number of times in the top and bottom peripheral boxes in each SOA. In each experimental block, there were 32 trials where the target was present and 8 trials where the target was absent, in order to discourage anticipated responses. In the present trials, there were 8 trials for each SOA condition. In half of these trials (4) the target was presented at the cued location, and it was presented at the uncued location in the remaining trials.

\section{Results and discussion}

Following the same criteria as Colzato et al. (2008), responses faster than $100 \mathrm{~ms}$ or slower than $900 \mathrm{~ms}$ were excluded from the analyses ( $3 \%$ of the trials). An ANOVA with Type of cue (valid vs. invalid) and SOA (100, $400,700,1000)$ as within-subject variables, and Group of Participants (monolingual vs. bilingual) as betweensubject variable, was conducted.

In the error analyses, a main effect of Type of cue $(F(1,54)=10.79, M S E=15.77, p<.002)$ was observed, reflecting worse performance for invalid (6.0\% errors) than for valid trials ( $4.8 \%$ errors). There was also a main effect of SOA $(F(3,162)=4.04, M S E=24.57, p<$ .008 ), revealing more errors at SOA 100 than at SOA 400 and 700 (all $p \mathrm{~s}<.03$ ). No other effects were significant $(F \mathrm{~S}<1)$.

The RT analyses revealed a main effect of SOA $(F(3,162)=7.64, M S E=521.28, p<.0001)$. The main effects of Type of cue and Group of Participants were not significant $(F \mathrm{~s}<1)$. The only significant interaction 
Table 2. PANEL A: Means of reaction times (RTS) as a function of Type of Cue (valid and invalid), SOAs (100, 400, 700 and 1000) and Group of Participants (bilinguals and monolinguals). PANEL B: Means of percentage of errors (\% errors) as a function of Type of Cue (valid and invalid), SOAs and Group of Participants. Values in brackets refer to standard errors.

\begin{tabular}{|c|c|c|c|c|c|c|c|c|}
\hline \multirow{2}{*}{$\begin{array}{l}\text { PANEL A. RTs } \\
\text { Group }\end{array}$} & \multicolumn{4}{|c|}{ Valid cue } & \multicolumn{4}{|c|}{ Invalid cue } \\
\hline & SOA 100 & SOA 400 & SOA 700 & SOA 1000 & SOA 100 & SOA 400 & SOA 700 & SOA 1000 \\
\hline Bilingual & $531(62.9)$ & $524(56.4)$ & $536(55.8)$ & $543(60.3)$ & $543(63)$ & $527(62.5)$ & $523(62.8)$ & $538(61.9)$ \\
\hline Monolingual & $521(58.9)$ & $518(59.9)$ & $523(70)$ & $529(64.6)$ & $536(59.3)$ & $518(56.6)$ & $511(63.6)$ & $521(64.6)$ \\
\hline \multirow{2}{*}{$\begin{array}{l}\text { PANEL B. \% errors } \\
\text { Group }\end{array}$} & \multicolumn{4}{|c|}{ Valid cue } & \multicolumn{4}{|c|}{ Invalid cue } \\
\hline & SOA 100 & SOA 400 & SOA 700 & SOA 1000 & SOA 100 & SOA 400 & SOA 700 & SOA 1000 \\
\hline Bilingual & $5.7(4.8)$ & $4.2(5.5)$ & $4.5(3.9)$ & $5.4(6.5)$ & $7.2(4.7)$ & $5.4(5)$ & $6.6(5.6)$ & $6.8(7.4)$ \\
\hline Monolingual & $5.4(6.1)$ & $3.9(3.7)$ & $5.2(6.8)$ & $4.2(4.3)$ & $7.9(6)$ & $3.7(4.3)$ & $4.4(3.9)$ & $6.5(6.5)$ \\
\hline
\end{tabular}

PANEL A. Data from our Experiment 2 Error bars represent standard errors

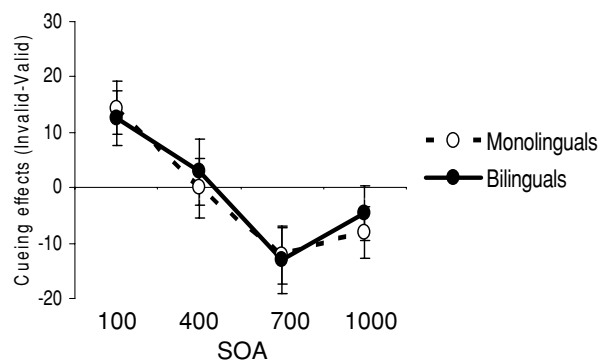

PANEL B. Data from Colzato et al. (2008) Error bars were not provided

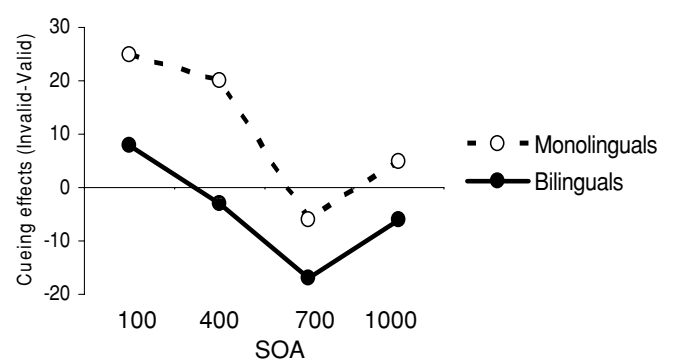

Figure 3. Magnitude of the cueing effects (in ms) as a function of SOA and Group of Participants.

was the one between Type of cue and SOA $(F(3,162)=$ 11.68, $M S E=302.49, p<.0001)$. This interaction revealed that at SOA 100 , RTs for valid trials were significantly faster $(13 \mathrm{~ms})$ than for invalid trials (the cueing facilitation effect; $t(55)=3.9, p<.0001$ ), while at SOA 700, RTs for valid trials were significantly slower $(14 \mathrm{~ms})$ than for invalid trials (the IOR effect; $t(55)=$ $3.18, p<.002$ ). No effects of Type of cue were observed in the other two SOAs (see Table 2 for a report of RTs and percentage of errors). Thus, facilitatory and inhibitory effects of valid cues were both present in this experiment.

What is fundamental for our purpose here is that none of the main effects (neither SOA nor Type of cue) interacted with the variable Group of Participants (all $F$ s $<1$ ). As can be easily appreciated in Figure 3 (Panel A), the magnitude of the cueing facilitation and inhibition (IOR) effects were exactly the same for bilinguals and monolinguals. This simple pattern clearly contrasts with the complex one reported by Colzato et al. (2008), in which bilinguals did not show the cueing facilitation effect and monolinguals did not show the cueing inhibition effect (see Figure 3 Panels A and B). Further, our observations ${ }^{5}$

\footnotetext{
5 We have additional evidence regarding the lack of a bilingual impact on cueing effects. In an experiment where the Numerical Stroop and the cueing paradigm were combined in a single task, bilinguals and monolinguals showed a similar magnitude of inhibitory cueing effects at a long SOA (bilinguals: $28 \mathrm{~ms}$, monolinguals: $34 \mathrm{~ms} ; F(1,60)<1$ ). This experiment was conducted by 32 young Catalan-Spanish bilinguals and 32 young Spanish monolinguals - two were removed from the analyses because of a very low score in the Raven test - from the same population as Experiment 2. Furthermore, in this experiment a reliable significant difference in the magnitude of the Stroop interference effect was observed between bilinguals and monolinguals ("Group of Participants" and "Stroop interference effect" (bilinguals: $27 \mathrm{~ms}$, monolinguals: $42 \mathrm{~ms} ; F(1,60)=5.62, M S E=588.5, p<.02)$. Hence, this experiment shows that it is possible to observe a bilingual advantage in conflict resolution in the same experimental context where no bilingual effect is observed on the orienting network. This pattern or results is consistent with our previous observations (Costa et al., 2008).
} 
are inconsistent with the conclusion reached by Colzato et al. ("it is clear that cueing effects were affected by bilingualism", p. 307). We find no significant effect of bilingualism on cueing effects.

\section{General discussion}

The main objective of the present article was to provide evidence regarding the effects of bilingualism on the attentional system. In Experiment 1, we explored the effects of bilingualism on the executive control network, by means of the Numerical Stroop Task. In Experiment 2, we assessed the effects of bilingualism on the orienting network through a detection task involving visual cues. The results of the two experiments alleviate concerns about the robustness and replicability of a bilingual advantage on executive control and delimit the scope of the impact of bilingualism on other components of the attentional system.

In a nutshell, three main results were observed in our experiments. First, bilinguals exhibited a reduced Stroop interference effect compared to monolinguals. Second, bilinguals experienced a larger Stroop facilitation effect than monolinguals. Third, the pattern of cueing effects was the same for both groups. We discuss the implications of these findings in turn.

\section{The effect of bilingualism on the executive network}

The observation of a bilingual advantage in the SIE fits well with previous observations revealing a benefit of bilingualism in tasks that involve conflict resolution (Bialystok et al., 2004, 2005a, b; Bialystok, 2006; Costa et al., 2008; Bilaystok et al., 2008). Our observation is important given the concerns that have been raised about the replicability of the advantage on conflict resolution in adult young bilinguals. The fact that we were not only able to find such a bilingual advantage, but also that we did so with a task that departs in some ways from those already used (mostly of spatial or linguistic nature), helps to increase our confidence on the reliability and generalizability of the bilingual impact on conflict resolution tasks.

As advanced in the "Introduction", the bilingual advantage in conflict resolution has often been attributed to the collateral effects of bilingual language control. In particular, it has been argued that bilinguals may resort to inhibitory control during language production in order to suppress the activation of the non-intended language (e.g., Green, 1998; Bialystok et al., 2004; Kroll et al., 2008). This mechanism would allow bilinguals to select the target word in the intended language while avoiding massive interference from alternative "incompatible" translation words. Moreover, such inhibitory processes may be the same as those implicated on resolving the conflict created by irrelevant incongruent stimuli. Hence, the continuous use of inhibitory processes by bilinguals during language production may result in the observed advantage in language-free conflict resolution tasks.

Although this explanation adequately captures a large body of experimental evidence, it also leaves several results unaccounted for. In particular, it does not account for the bilingual effect in conditions in which conflict resolution is not needed. For example, in our Experiment 1, the magnitude of the SFE was modulated by bilingualism: monolinguals took advantage of congruent irrelevant stimuli to a lesser extent than bilinguals (see also Bialystok et al., 2008). Arguably, in this condition inhibitory processes are not necessary since the two dimensions (the target and the distractor) call for the same response. Why then do bilinguals outperform monolinguals in these conditions? One possibility is that bilingualism has an impact on some aspects of executive control other than inhibitory processes such as those responsible for adjusting or regulating behavior (i.e., monitoring processes; see Roelofs, van Turennout and Coles (2006) for a pertinent discussion of this possibility in the light of neuroimaging data). Under this view, the larger SFE for bilinguals may be interpreted as revealing a more efficient functioning of this regulative process involved in response selection. That is, bilinguals may be better not only at inhibiting competing conflicting responses, but also at boosting the correct response according to the task demands; hence, their larger SFE. Admittedly, this is a tentative explanation that needs further research. Nevertheless, what this result reveals is a bilingual effect on conditions in which conflict resolution is not required, suggesting an effect of bilingualism beyond inhibitory processes.

\section{The impact of bilingualism on the orienting network}

In the "Introduction", we argued that one way to explore the origin of the bilingual advantage in attentional processes is to advance in our knowledge of its limits. We further argued that given the orchestrated manner in which the different attention networks work, it is conceivable that the effects of bilingualism on the executive network percolate, to some extent, to the other networks and, in particular, to the orienting network. Given that the available results regarding the effects of bilingualism on the orienting network of attention were mixed (Colzato et al., 2008; Costa et al., 2008), it was fundamental to further explore this issue.

Unlike the complex pattern of findings in the Colzato et al. (2008) study, where cueing effects interacted with group of participants, Experiment 2 found that the magnitude or time course of benefits and costs of visual cues was unaffected by bilingualism. A closer look at the whole pattern of results reveals important differences between the two studies. First, in Colzato et al.'s study, the benefit produced by valid cues at short SOAs seemed 
to be present only for monolinguals (while in our study, it was present for both groups and to the same extent). Second, the cueing inhibition effect produced by valid cues at long SOAs was only present for bilinguals in Colzato et al. (while in our study, it was present for both groups and to the same extent). Given that previous studies have consistently found a cueing facilitation effect for bilinguals (Costa et al., 2008) and cueing inhibition effect for monolinguals (see Lupiáñez, Milliken, Solano, Weaver and Tipper, 2001), the source of the group variability in Colzato et al.'s study is surprising and it is not accounted for by the authors. Interestingly, however, if one averages the performance of bilinguals and monolinguals in their study, the results remarkably mimic the ones reported in our Experiment 2, namely cueing facilitation and inhibition effects for both monolinguals and bilinguals.

Several differences between the two studies, in terms of the populations tested, seemed to not be able to account for the different pattern of results. Both groups of bilinguals were early and high-proficient bilinguals that used the two languages on daily basis. Although Colzato et al. compare bilinguals and monolinguals of different national backgrounds (Dutch-German or Dutch-English bilinguals vs. Spanish monolinguals), while we tested groups of the same national background (Catalan-Spanish bilinguals living in Barcelona and Spanish monolinguals living in Murcia, $500 \mathrm{~km}$ from Barcelona), such differences are unlikely to account for the different pattern of results. Finally, IQ values for the two groups in Colzato et al.'s Experiment 2 are not reported, but a difference in IQ values between the two groups seems unlikely.

At this point, and given the available results (Experiment 2; see also footnote 4 and Costa et al., 2008) it appears that little evidence is present to conclude that the cueing effects for monolinguals and bilinguals are different. Thus, we have good grounds to conclude that the attentional orienting processes with exogenous cues that depend on the orienting network are not affected by bilingualism.

\section{Conclusion}

The results reported in this article help to constrain the potential effect of bilingualism on the attentional system in two relevant ways. First, they provide new evidence revealing that bilingualism exerts an effect in the executive control network (at least as it is indexed by conflict resolution tasks). Second, they strongly suggest that bilingualism does not exert any effect in the processes involved in the orienting network of attention (at least as it is indexed by cueing effects). Future research is required to determine which of the processes under the umbrella of executive control are indeed being modulated by bilingualism.

\section{Appendix. Description of participants from all experiments}

Bilingual and monolingual participants in all three experiments differed in their language history. The monolinguals of all experiments were Spanish and were Psychology undergraduates in the University of Murcia (Spain). The bilinguals of all experiments were CatalanSpanish early and high-proficient bilinguals and were Psychology undergraduates in the University of Barcelona (Spain). All participants were young individuals ranging from seventeen to thirty years. Bilinguals and monolinguals did not differ in age either in Experiment 1 (bilinguals: 20.9 years, monolinguals: 21.4 years; $t(80)=$ 0.92, $p<.36$ ) or in Experiment 2 (bilinguals: 20.2, monolinguals: $20.9 ; t(54)=1.25, p<.21)$.

All bilinguals were exposed to two languages at a very early age and received their education in the two languages. Information about bilinguals' language use was obtained by means of a questionnaire administered after the experiment, where scores represent the amount of the time participants used each language, indicated on a 7 -point scale $(1=$ only Spanish, $7=$ only Catalan $)$. Mean scores showed that they have used Catalan around $75 \%$ of time and Spanish around $25 \%$ across their lifespan (Experiment 1: 5.1 in preschool age, 5.2 in childhood, 5.1 in adolescence and 5.1 in adulthood; Experiment 2: 5.1 in preschool age, 5.2 in childhood, 5 in adolescence and 4.9 in adulthood). Neither bilinguals nor monolinguals were functionally fluent in any other language despite foreign language instruction in school. Importantly, specific care was taken to match both groups of the three experiments in general intelligence and video-game experience.

General intelligence was assessed by means of the Superior Scale I of the Raven's Advanced Progressive Matrices (Raven, Raven and Court, 1998), which participants completed after the experimental session. The task was composed of 12 items containing a picture with a missing piece. Participants were asked to indicate which of the 8 possible pieces arranged below the picture completed it correctly. Both groups of participants were comparable in the scores obtained in this task in Experiment 1 (bilinguals: 10.7, monolinguals: 10.6; $t(80)=$ $0.24, p<.81$; which, according to Spanish normative data (Seisdedos, 1995), means that both groups had as an IQ average of 114), and Experiment 2 (bilinguals: 10.9, monolinguals: $10.6 ; t(54)=1.08, p<.29$; which, according to Spanish normative data (Seisdedos, 1995), means that both groups had as an IQ average of 114). Also, all participants had conducted a common mandatory exam to be enrolled in university. No differences between groups were observed in the grades obtained on this exam either in Experiment 1 (bilinguals: 6.8, monolinguals: 6.7; $t(80)=0.89, p<.37$ ) or Experiment 2 (bilinguals: 6.7, monolinguals: $6.6 ; t(54)<1)$. 
An additional questionnaire was administered to all participants to determine the level of computer video-game experience (see Green and Bavelier, 2003). Participants specified the extent to which they played speeded video games in the previous six months. They were asked to estimate the time they spend playing computer video-games per week a on a 4-point scale $(0=$ none, $4=$ daily $)$. Participants also indicated how many hours they spend playing video-games in those days they play, on a 4-point scale $(0=$ none, $4=$ more than three hours). The total score is the sum of Times/week and Time/day for each subject. No significant differences between groups were found in computer video-game experience either in Experiment 1 (bilinguals: 1.02 , monolinguals: $1.41 ; t(80)=1.11, p<$ 0.27 ), or Experiment 2 (bilinguals: 1, monolinguals: 1.41; $t(54)<1)$.

\section{References}

Abutalebi, J. \& Green, D. (2007). Bilingual language production: The neurocognition of language representation and control. Journal of Neurolinguistics, 20, 242-275.

Abutalebi, J. \& Green, D. (2008). Control mechanisms in bilingual language production: Neural evidence from language switching studies. Language and Cognitive Processes, 23 (4), 557-582.

Barceló, F. (2003). The Madrid card sorting test (MCST): A task switching paradigm to study executive attention with eventrelated potentials. Brain Research Protocols, 11, 27-37.

Bialystok, E. (2006). Effect of bilingualism and computer video game experience on the Simon task. Canadian Journal of Experimental Psychology, 60 (1), 68-79.

Bialystok, E., Craik, F. I. M., Grady, C., Chau, W., Ishii, R., Gunji, A. et al. (2005b). Effect of bilingualism on cognitive control in the Simon task: Evidence from MEG. NeuroImage, 24, 40-49.

Bialystok, E., Craik, F. I. M., Klein, R. \& Viswanathan, M. (2004). Bilingualism, aging, and cognitive control: Evidence from the Simon task. Psychology and Aging, 19, 290-303.

Bialystok, E., Craik, F. I. M. \& Luk, G. (2008). Cognitive control and lexical access in younger and older bilinguals. Journal of Experimental Psychology: Learning, Memory, and Cognition, 34 (4), 859-873.

Bialystok, E., Martin, M. M. \& Viswanathan, M. (2005a). Bilingualism across the lifespan: The rise and fall of inhibitory control. International Journal of Bilingualism, 9 (1), 103-119.

Callejas, A., Lupiáñez, J., Funes, M. J. \& Tudela, P. (2005). Modulations among the alerting, orienting and executive control networks. Experimental Brain Research 167, 27 37.

Callejas, A., Lupiáñez, J. \& Tudela, P. (2004). The three attentional networks: On its independence and interactions. Brain and Cognition, 54, 225-227.

Colzato, L. S., Bajo, M. T., van den Wildenberg, W., Paolieri, D., Nieuwenhuis, S., La Heij, W. \& Hommel, B. (2008).
How does bilingualism improve executive control? A comparison of active and reactive inhibition mechanisms. Journal of Experimental Psychology: Learning, Memory, and Cognition, 34 (2), 302-312.

Costa, A. \& Caramazza, A. (1999). Is lexical selection in bilingual speech production language-specific? Further evidence from Spanish-English and English-Spanish bilinguals. Bilingualism: Language and Cognition, 2, 231244.

Costa, A., Hernández, M., Costa-Faidella, J. \& SebastiánGallés, N. (2009). On the bilingual advantage in conflict processing: Now you see it, now you don't. Cognition, 113, 135-149.

Costa, A., Hernández, M. \& Sebastián-Gallés, N. (2008). Bilingualism aids conflict resolution: Evidence from the ANT task. Cognition, 106, 59-86.

Costa, A., Miozzo, M. \& Caramazza, A. (1999). Lexical selection in bilinguals: Do words in the bilingual's two lexicons compete for selection? Journal of Memory and Language, 41, 365-397.

Costa, A., Santesteban, M. \& Ivanova, I. (2006). How do highlyproficient bilinguals control their lexicalization process? Inhibitory and language-specific selection mechanisms are both functional. Journal of Experimental Psychology: Learning, Memory and Cognition, 32, 1057-1074.

Fan, J., McCandliss, B. D. \& Fossella, J. (2005). The activation of attentional networks. Neurolmage, 26, 471-479.

Fan, J., McCandliss, B. D., Sommer, T., Raz, A. \& Posner, M. I. (2002). Testing the efficiency and independence of attentional networks. Journal of Cognitive Neuroscience, 14, 340-347.

Finkbeiner, M., Gollan, T. H. \& Caramazza, A. (2006). Lexical access in bilingual speakers: What's the (hard) problem? Bilingualism: Language and Cognition, 9, 153-166.

Fuentes, L. J. \& Campoy, G. (2008). The time course of alerting eVect over orienting in the attention network test. Experimental Brain Research, 185 (4), 667-672.

Green, C. \& Bavelier, D. (2003). Action video game modifies visual selective attention. Nature, 423, 534-537.

Green, D. W. (1986). Control, activation and resource. Brain and Language, 27, 210-223.

Green, D. W. (1998). Mental control of the bilingual lexicosemantic system. Bilingualism: Language and Cognition, $1,67-81$.

Kroll, J. F., Bobb, S. C., Misra, M. \& Guo, T. (2008). Language selection in bilingual speech: Evidence for inhibitory processes. Acta Psychologica, 128, 416-430.

Kroll, J. F., Bobb, S. C. \& Wodniecka, Z. (2006). Language selectivity is the exception, not the rule: Arguments against a fixed locus of language selection in bilingual speech. Bilingualism: Language and Cognition, 9 (2), 111135.

Lee, M. W. \& Williams, J. N. (2001). Lexical access in spoken word production by bilinguals: Evidence from the semantic competitor priming paradigm. Bilingualism: Language and Cognition, 4, 233-248.

Lupiáñez, J., Milliken, B., Solano, C., Weaver, B. \& Tipper, S. (2001). On the strategic modulation of the time course of facilitation and inhibition of return. Quarterly Journal of Experimental Psychology, 54A, 753-773. 
Martin-Rhee, M. M. \& Bialystok, E. (2008). The development of two types of inhibitory control in monolingual and bilingual children. Bilingualism: Language and Cognition, 11 (1), 81-93.

Meuter, R. F. I. \& Allport, A. (1999). Bilingual language switching in naming: Asymmetrical costs of language selection. Journal of Memory and Language, 40, 25-40.

Milliken, B., Lupiáñez, J. \& Roberts, M. (2003). Orienting in space and time: Joint contributions to exogenous spatial cueing effects. Psychonomic Bulletin \& Review, 10 (4), 877-883.

Morton, J. B. \& Harper, S. N. (2007). What did Simon say? Revisiting the bilingual advantage. Developmental Science, 10 (6), 719-726.

Posner, M. I. \& Boies, S. J. (1971) Components of attention. Psychological Review, 78 (5), 391-408.

Posner, M. I. \& Petersen, S. E. (1990). The attention system of the human brain. Annual Review of Neuroscience, 13, $25-42$.

Raven, J., Raven, J. C. \& Court, J. H. (1998). Manual for Raven's Advanced Progressive Matrices (1998 edn). Oxford: Oxford Psychologists Press.
Roelofs, A. (1998). Lemma selection without inhibition of languages in bilingual speakers. Bilingualism: Language and Cognition, 1, 9495.

Roelofs, A., van Turennout, M. \& Coles, M. G. H. (2006). Anterior cingulate cortex activity can be independent of response conflict in Stroop-like tasks. Proceedings of the National Academy of Sciences, 103 (37), 1388413889.

Ruz, M. \& Lupiáñez, J. (2002). A review of Attentional Capture: On its automaticity to endogenous control. Psicológica, 23, 283-309.

Seisdedos, N. (1995). Matrices progresivas de Raven: Escalas: CPM color, SPM general, APM superior. Madrid: TEA.

Simon, J. R. \& Rudell, A. P. (1967). Auditory S-R compatibility: The effect of an irrelevant cue on information processing. Journal of Applied Psychology, 51, 300 304.

Stroop, J. R. (1935). Studies of interference in serial verbal reactions. Journal of Experimental Psychology, 18, 643662. 